\title{
Structural study of aged saturated silica gels obtained from tetramethoxysilane sonohydrolysis with different water/tetramethoxysilane molar ratio
}

\author{
D. R. Vollet, D. A. Donatti, A. Ibañez Ruiz, and W. C. de Castro \\ Department of Physics, IGCE-UNESP, POB 178 CEP 13500-970 Rio Claro (SP), Brazil
}

(Received 28 November 2002; published 30 May 2003)

\begin{abstract}
The structural characteristics of saturated silica sonogels were studied by means of small-angle x-ray scattering (SAXS) and thermogravimetric analysis (TG), after a long time of aging in saturated conditions. The sonogels were obtained by a sol-gel routine from ultrasound stimulated tetramethoxysilane (TMOS) hydrolysis carried out with the initial water/TMOS molar ratio $(r)$ ranging from 2 to 10 . The saturated sonogel structure can be described as composed by mass fractal-like aggregates (clusters) of primary silica particles, all imbibed in a liquid phase. The values of the mass fractal dimension $(D)$ of the clusters was found all around 2.5 , while the characteristic size of the clusters $(\xi)$ was found generally increasing with $r$, going from approximately 2.3 $\mathrm{nm}(r=2)$ to $4.5 \mathrm{~nm}(r=10)$. The volume fraction of the clusters was estimated from the SAXS data. The results were compared to the values of weight loss fraction at the inflection point that has been found in the derivative of the TG curve, which should accounts for the instant in which the meniscus of the liquid phase penetrates into the clusters under a rapid evaporation process as in a TG test.
\end{abstract}

DOI: 10.1103/PhysRevB.67.184207

PACS number(s): 61.10.Eq, 61.43.Hv, 81.20.Fw, 82.70.Gg

\section{INTRODUCTION}

A large variety of glass and glass ceramic has been obtained by sol-gel process from the hydrolysis of tetraalkoxysilanes. ${ }^{1}$ Since water and alkoxide frequently exhibit an immiscibility gap, a co-solvent such as alcohol is used as a homogenizing medium for the hydrolysis process (conventional method). Ultrasound is an alternative method to promote hydrolysis of alkoxide without using alcoholic co-solvent for homogenization (sonohydrolysis). ${ }^{2}$ However, there is no simple criterion to be established for the equivalence in the sample preparation to allow a direct comparative study between the both methods. For instance, starting with a fixed quantity of alkoxide and fixing the total volume of the mixture, we could not find an adequate relation between water and co-solvent in the conventional method which matches the same water/alkoxide molar ratio necessary to the solventless sonohydrolysis.

It has been generally accepted that fractal structures are formed in the sol-gel process. ${ }^{3}$ However, different fractal structures have been reported depending on the conditions of preparation, such as the base or acid-catalyzed hydrolysis, the aging time before the supercritical treatment (in the case of aerogels) and the initial alkoxide/water molar ratio. ${ }^{4}$ Since the structure and properties of the final product have been found to be strongly dependent of the initial conditions of preparation, thus the structural properties of sonogels have been subject to some speculation. Some studies on the time evolution about the aggregation process ${ }^{5}$ and the aging process $^{6}$ have been carried out in sonogels. Indeed, the literature is not too plentiful of data concerning to the structural properties of sonogels, mainly about saturated sonogels.

In this work, the structural characteristics of tetramethoxysilane (TMOS)-derived sonogels, after a long time of aging in saturated conditions, are studied as a function of the starting water/TMOS molar ratio used in the sonohydrolysis. The saturated sonogels were analyzed by small-angle x-ray scat- tering (SAXS) and thermogravimetry (TG). An interesting structural correlation obtained from SAXS and TG data for the volume fraction of the clusters composing the saturated gels is presented.

\section{EXPERIMENT}

\section{A. Sample preparation}

Tetramethoxysilane (TMOS), distilled and deionized water, and oxalic acid as a catalyst were used to prepare acidulated TMOS-water mixtures of constant volume $(31 \mathrm{ml})$ with $0.116 \mathrm{~mol} / \mathrm{l}$ oxalic acid and water/TMOS molar ratio $(r)$ ranging from 2 to 10 . The samples were submitted to a constant power $(60 \mathrm{~W})$ of ultrasonic radiation using a $20-\mathrm{kHz}$ $600-\mathrm{W}$ apparatus, with a 13 -mm-diameter titanium transducer driven by an electrostrictive device. As described elsewhere, ${ }^{7}$ a stainless-steel reaction cell is held under a thermostatic temperature $\left(25^{\circ} \mathrm{C}\right)$ by means of high flux water circulation from an external bath. The acid action is mainly associated to the hydrolysis process, which occurs rapidly and completely at the first instants of the sonication process. ${ }^{8}$ The resulting sols were held at room temperature in perfectly sealed containers to gelation and aging under completely saturated conditions for about a year. A long time of aging is desired since we aim to study the structure after practically complete polycondensation reactions, when no more structural evolution is expected to occur and the effect of the initial water quantity on the final structure can be probed. We could accelerate the polycondensation process by the addition of $\mathrm{NH}_{4} \mathrm{OH}$, for instance. However, the use of a two-step process would bring a new variable to be considered and the effect of the water content under probe could be influenced. The aged monolithic sonogels were studied by SAXS, bulk density, and termogravimetry analysis.

\section{B. SAXS measurements}

The SAXS experiments were carried out using synchrotron radiation with a wavelength $\lambda=0.1608 \mathrm{~nm}$. The beam 
was monochromatized by a silicon monochromator and collimated by a set of slits defining a pin-hole geometry. A one-dimensional (1D) position sensitive x-ray detector was used to record the SAXS intensity as function of the modulus of the scattering vector $q=(4 \pi / \lambda) \sin (\theta / 2)$, where $\theta$ is the scattering angle. The experimental set allowed us to obtain SAXS data from $q_{0}=0.1 \mathrm{~nm}^{-1}$ to $q_{m}=3.5 \mathrm{~nm}^{-1}$ with a resolution of $\Delta q=4.98 \times 10^{-3} \mathrm{~nm}^{-1}$. Samples of about 1 $\mathrm{mm}$ thick were cut from the saturated sonogels for the SAXS experiments. The samples were never allowed to dry during the SAXS measurements. The SAXS data were corrected by the parasitic scattering and the sample attenuation, and normalized by incident beam intensity and the logarithm of the attenuation, which is fairly proportional to the thickness of the sample, if we neglect the variations of composition due to the different water contents.

\section{Interpretation of SAXS data}

An approach suggested by Freltoft et al. ${ }^{9}$ which has been used ${ }^{10}$ to describe the SAXS intensity $I(q)$ from a system of mass fractal-like aggregates (clusters) of primary particles can be expressed by

$$
I(q)=A P(q) S(q),
$$

where $A$ is a parameter describing the independent scattering from all primary particles, $P(q)$ the form factor of a single particle, and $S(q)$ the structure factor of the clusters. The parameter $A$ is proportional to the number of primary particles in irradiated volume, $P(q)$ is often well approximated by the Debye-Bueche form

$$
P(q)=1 /\left(1+a_{1}^{2} q^{2}\right)^{2},
$$

where $a_{1}$ is the characteristic length of the primary particle and $S(q)$ is determined by

$$
\begin{gathered}
S(q)=1+B \sin [(D-1) \arctan (q \xi)] / \\
\left(1+q^{2} \xi^{2}\right)^{(D-1) / 2}(D-1) q \xi,
\end{gathered}
$$

where $\xi$ is the characteristic length of the cluster with mass fractal dimension $D$ and $B$ is a constant, so that $1+B$ represents an average number of primary particles per cluster.

For small values of $q$ so that $q \xi \ll 1$ the cluster seems to be homogeneous (nonfractal) to SAXS and the system should scatter at small $q$ values as Guinier's law as

$$
I(q)=I(0) \exp \left(-\frac{1}{3} R_{\mathrm{G}}^{2} q^{2}\right)
$$

where $R_{\mathrm{G}}$ is the gyration radius of the clusters, which is proportional to $\xi$. Very elongated clusters or a linear arrangement of minor globular clusters, which can be described as a cylindrical particle with a very long dimension $L$ compared to the cross-section dimensions, so that $q L \gg 1$ in Guinier's law region, should scatter at small $q$ as

$$
I(q) q=I_{\mathrm{c}}(0) \exp \left(-\frac{1}{2} R_{\mathrm{c}}^{2} q^{2}\right)
$$

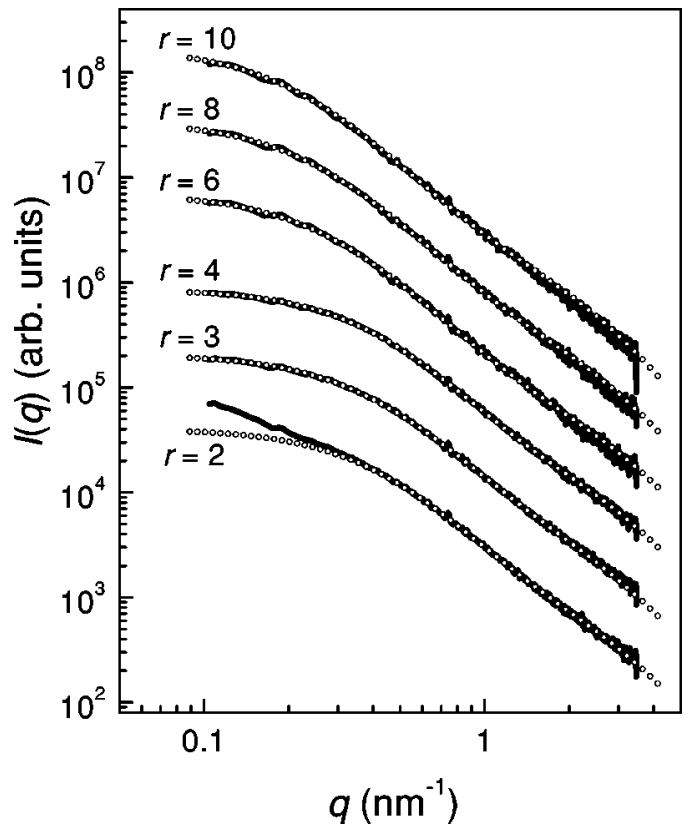

FIG. 1. SAXS intensity for saturated gels prepared with different initial water/TMOS molar ratio $(r)$. The intensities are multiplied by different factors in order to separate the curves. The dotted lines show the fitting of Freltoft et al. approach [Eqs. (1)-(3)] to the experimental data.

where $R_{\mathrm{c}}$ is the gyration radius of the cross section of the particle and $I_{\mathrm{c}}(q)$ the exclusive scattering of the cross section of the particle.

For high values of $q$ so that $q a_{1} \gg 1$, we expected the intensity going to a Porod's law behavior $I(q) \sim q^{-4} .{ }^{11} \mathrm{~Pa}$ rameter $a_{1}$ was found to be very small for Porod's law to be observed in the length scale probed by SAXS in this study. As a conservative estimative we assume that $a_{1} \sim 1 / q_{m}$ $\sim 0.3 \mathrm{~nm}$.

\section{RESULTS}

Figure 1 shows the experimental SAXS intensity as a function of the modulus of the scattering vector $q$ for saturated gels obtained from the sonohydrolysis of TMOS carried out with different water/TMOS molar ratio $(r)$. Freltoft et al.'s approach [Eqs. (1)-(3)] fits very well with the experimental SAXS data in all cases, except at low $q$ values for the sonogel prepared with $r=2$, in which case the fitting process was carried out in the range $q>0.35 \mathrm{~nm}^{-1}$. The fitting process was carried out by an iterative least-square routine with restriction in the parameter $a_{1}$ [Eq. (2)] so that $a_{1}$ $\sim 0.3 \mathrm{~nm}$, as pointed out in Sec. IIC. Parameters $D, \xi, B$, and $A$ of the fitting process are shown in Fig. 2 as a function of water/TMOS molar ratio.

The values of the mass fractal dimension $D$ were found all around $\sim 2.5$, mainly for large $r$ values. The average value for $D$ in the whole $r$ range was found to be $2.51 \pm 0.04$. The characteristic length of the clusters $\xi$ increases with the water quantity going from approximately $2.3 \mathrm{~nm}$ for $r=2$ to 4.5 $\mathrm{nm}$ for $r=10$, in agreement with the increase of the number of particle per cluster, $B+1$. It is apparent that there is some 


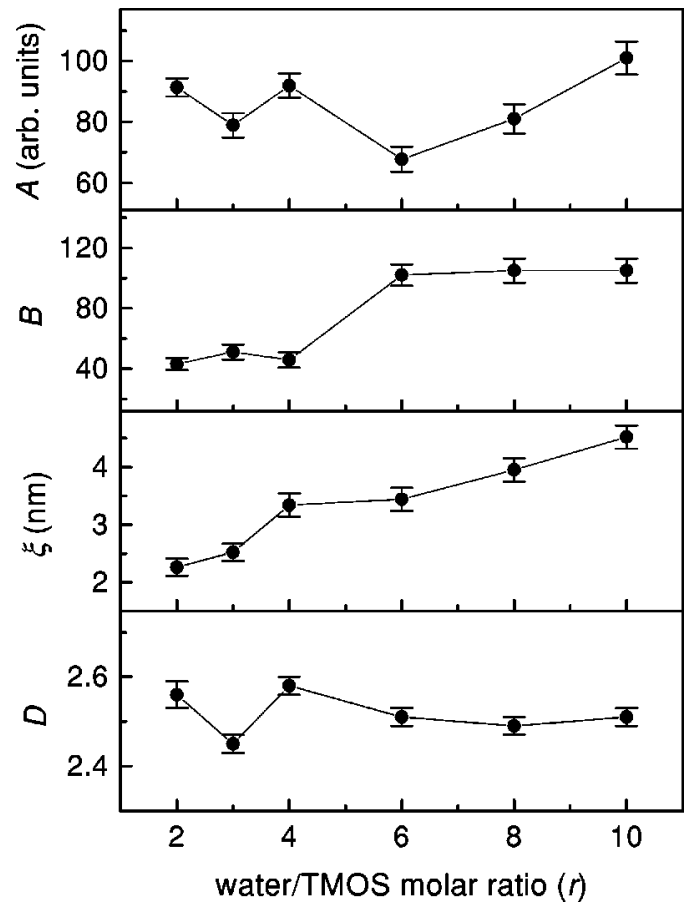

FIG. 2. Structural parameters as obtained by fitting Freltoft et al. approach [Eqs. (1)-(3)] to the experimental SAXS data as a function of the initial water/TMOS molar ratio.

discontinuity in the progress of the number of particles per cluster at around $r \sim 4$.

Figure 3 shows Guinier's plots as stated by Eqs. (4) and (5) at small $q$ values for some sonogels. From Fig. 4 (top) we can infer that Eq. (4) is not obeyed by the system at the

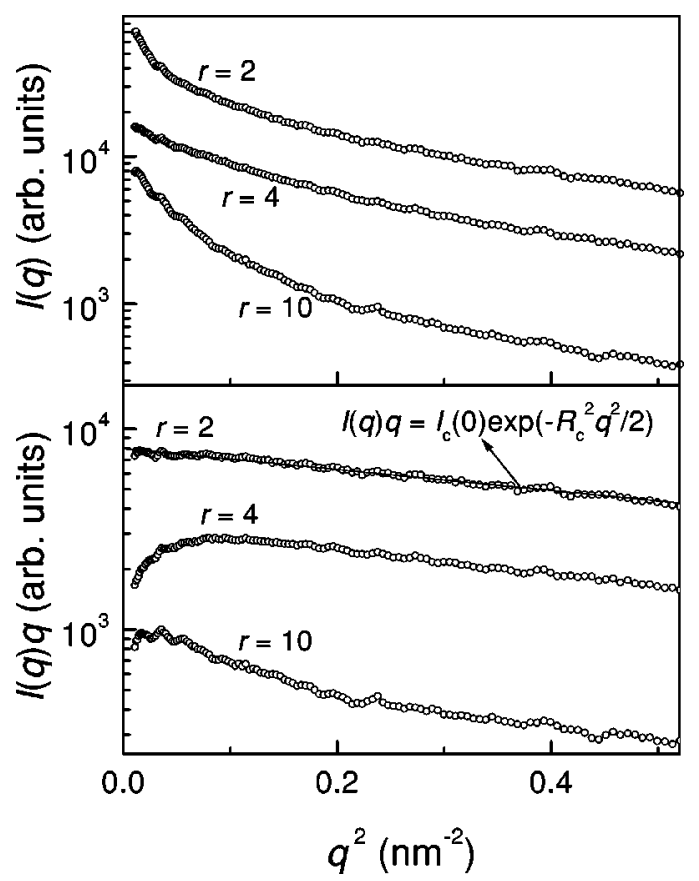

FIG. 3. Guinier's law plots as stated by Eqs. (4) (top) and (5) (bottom) for small $q$ values for several values of the initial water/ TMOS molar ratio $(r)$.

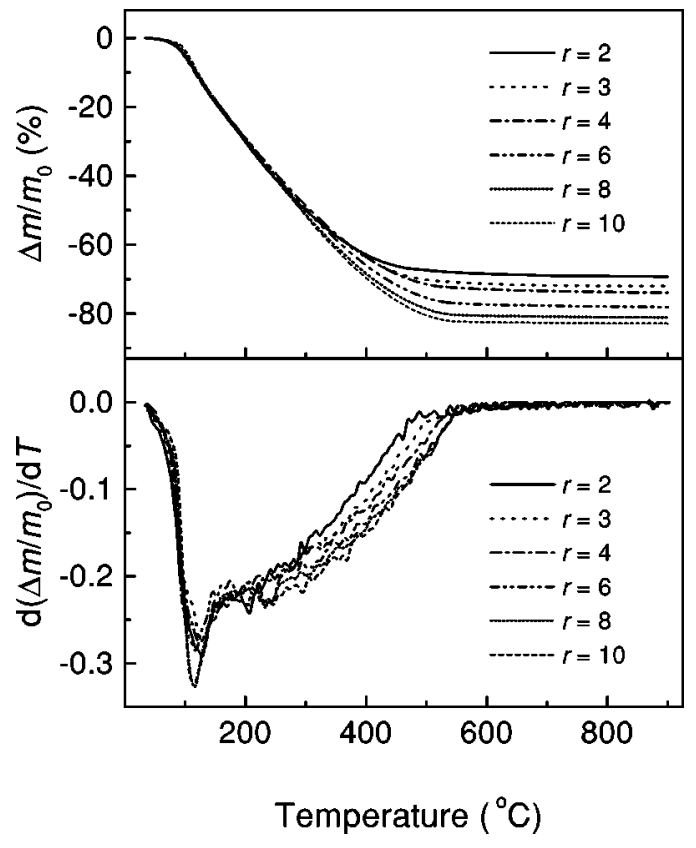

FIG. 4. Top: Temperature weight loss from saturated sonogels as determined by thermogravimetric analysis under a heating rate of $12.5^{\circ} \mathrm{C} \mathrm{min}^{-1}$ as a function of the initial water/TMOS molar ratio. Bottom: corresponding first derivative of the above curves.

extremes of water content $r=2$ and $r=10$, but is fairly obeyed for $r=4$. This means that the size distribution of clusters is more homogenous for $r=4$ when compared to that of $r=10$. The departure from Eq. (4) of the system with $r=2$ is due to another cause. From Fig. 3 (bottom) we can infer that Eq. (5) is well obeyed by the system with $r=2$. This means that probably the clusters are in a linear alignment so that they scatter, at this resolution scale, as a long cylindrical particle with a cross section with dimensions of the same order of the individual globular clusters to give a SAXS contribution as predicted by Eq. (5). The gyration radius of the cross section $R_{\mathrm{c}}$ for the sample with $r=2$, as determined by the linear fitting shown in Fig. 3 (bottom), was found to be $(1.53 \pm 0.02) \mathrm{nm}$. A cylinder with $R_{\mathrm{c}}$ $=1.53 \mathrm{~nm}$ has a cross-section radius equal to $2.16 \mathrm{~nm}$, which is in good agreement with the value $\xi=2.26 \mathrm{~nm}$ that was found by fitting the Freltoft et al. approach [Eqs. (1)(3)] to the sample with $r=2$.

Figure 4 (top) shows the weight loss percentage $\Delta m / m_{0}$ as determined by thermogravimetric analysis carried out under a heating rate of $12.5^{\circ} \mathrm{C} / \mathrm{min}$ up to $900{ }^{\circ} \mathrm{C}$, for saturated sonogels as a function of the initial water/TMOS molar ratio. Figure 4 (bottom) shows numerical first derivatives of the weight loss curves with respect to temperature $T$, $d\left(\Delta m / m_{0}\right) / d T$.

The total accumulative weight loss up to $900^{\circ} \mathrm{C}$, $\left(\Delta m / m_{0}\right)_{\mathrm{T}}$, was found increasing with the water content and the results are shown in Fig. 5 (bottom). For example, $\left(\Delta m / m_{0}\right)_{\mathrm{T}}$ was found to be about $69 \%$ for $r=2$ and about $84 \%$ for $r=10$. These results are in good agreement with those theoretical values (68\% and $82 \%$, respectively) estimated under the assumption of $100 \%$ hydrolysis followed (after a long time of aging) by $100 \%$ polycondensation re- 


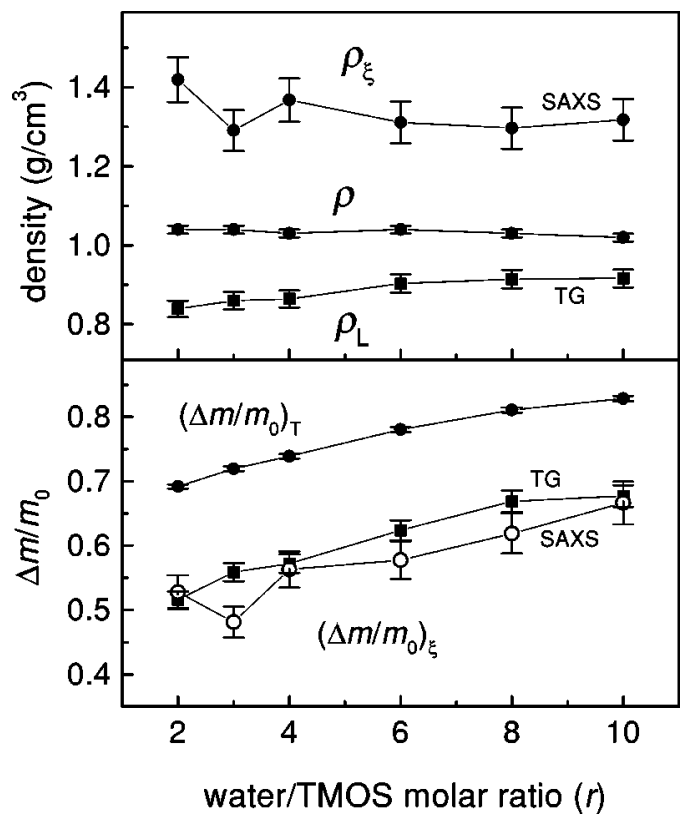

FIG. 5. Top: Densities associated to the saturated sonogels as a function of the initial water/TMOS molar ratio. $\rho$ : bulk density; $\rho_{\mathrm{L}}$ : density of the liquid phase of the sonogel; $\rho_{\xi}$ : cluster density. Bottom: $\left(\Delta m / m_{0}\right)_{\mathrm{T}}$ : total weight loss fraction as determined by TG; $\left(\Delta m / m_{0}\right)_{\xi}$ : weight loss fraction associated to the volume fraction of the clusters in the gels as determined by both TG and SAXS.

actions, under the condition of no liquid evaporation (since the containers are sealed), yielding exclusively $\mathrm{SiO}_{2}$, methanol and water (the latter when in excess in the case of $r$ $>2$ ). Under these conditions, the stoichiometry of the final sonogel could be cast as a mixture of $\mathrm{SiO}_{2}+4 \mathrm{CH}_{3} \mathrm{OH}+(r$ -2) $\mathrm{H}_{2} \mathrm{O}$, the liquid phase being a mixture of $4 \mathrm{CH}_{3} \mathrm{OH}$ $+(r-2) \mathrm{H}_{2} \mathrm{O}$. The bulk density of the samples $\rho$ were determined from mass and volume measurements and the results are shown in Fig. 5 (top). The liquid phase density $\rho_{\mathrm{L}}$ was evaluated from the total weight loss $\left(\Delta m / m_{0}\right)_{\mathrm{T}}$ and $\rho$ assuming that

$$
\rho=f_{\mathrm{L}} \rho_{\mathrm{L}}+\left(1-f_{\mathrm{L}}\right) \rho_{\mathrm{a}},
$$

where $f_{\mathrm{L}}$ is the volume fraction of the liquid phase in the gel and $\rho_{\mathrm{a}}$ is the density of primary particle composing the solid phase of the gel. Then

$$
\rho_{\mathrm{L}}=\left(\Delta m / m_{0}\right)_{\mathrm{T}} \rho_{\mathrm{a}} /\left\{\left(\rho_{\mathrm{a}} / \rho\right)-\left[1-\left(\Delta m / m_{0}\right)_{\mathrm{T}}\right]\right\} .
$$

Figure 5 (top) shows the evaluated values for $\rho_{\mathrm{L}}$ assuming $\rho_{\mathrm{a}}$ equal to the density of the fused silica $2.25 \mathrm{~g} / \mathrm{cm}^{3}$. The values of $\rho_{\mathrm{L}}$ were found all about $6 \%$ less than those theoretical evaluated from an ideal liquid mixture with composition 4 $\mathrm{CH}_{3} \mathrm{OH}+(r-2) \mathrm{H}_{2} \mathrm{O}$.

\section{DISCUSSION}

The structure of the TMOS-derived sonogels after a long period of aging in perfectly sealed containers can be described as formed by mass fractal-like aggregates (clusters) of primary particles of silica, all imbibed in a liquid phase.
The mass fractal dimension of the clusters was found to be about 2.5. A value of $D \sim 2.5$ (together with an exponent of polydispersity $\tau \sim 2.2$ ) would be compatible with a aggregation process according to the percolation theory. ${ }^{12}$

The characteristic length of the clusters and their structural arrangement seem to be a function of the initial water/ TMOS molar ratio used in the sonohydrolysis process. For instance, for $r=2$ the clusters are strongly aligned forming long chains with a sharp distribution of size in the characteristic cross-section dimension, while for $r=10$ the distribution of size of the globular clusters is wider.

The density $\rho_{\xi}$ of a cluster with characteristic length $\xi$ and mass fractal $D$, composed by particles of characteristic length $a_{1}$ and density $\rho_{\mathrm{a}}$, all imbibed in a liquid phase with density $\rho_{\mathrm{L}}$, can be evaluated from the following equation: ${ }^{13}$

$$
\Delta \rho_{\xi} / \Delta \rho_{\mathrm{a}}=\left(\xi / a_{1}\right)^{D-3},
$$

where $\Delta \rho_{\xi}=\rho_{\xi}-\rho_{\mathrm{L}}$ and $\Delta \rho_{\mathrm{a}}=\rho_{\mathrm{a}}-\rho_{\mathrm{L}}$. Figure 5 (top) shows the values of the density of the clusters $\rho_{\xi}$ as a function of the initial water/TMOS molar ratio, as evaluated from Eq. (8) with $\rho_{\mathrm{a}}=2.25 \mathrm{~g} / \mathrm{cm}^{3}$ and the data of $\rho_{\mathrm{L}}$ shown in Fig. 5 and the SAXS parameters $\xi$ and $D$ shown in Fig. 2. The volume fraction of the clusters in the sonogels, $f_{\xi}$, can be evaluated from an analogy to Eq. (6) by writing

$$
\rho=f_{\xi} \rho_{\xi}+\left(1-f_{\xi}\right) \rho_{\mathrm{L}} .
$$

Then

$$
f_{\xi}=\left(\rho-\rho_{\mathrm{L}}\right) /\left(\rho_{\xi}-\rho_{\mathrm{L}}\right) .
$$

For instance, the evaluation of Eq. (10) for the sample with $r=4$ yields $f_{\xi}=0.329$. Thus we presume that about 0.671 of the volume fraction of the sonogel is associated to a macroporosity occupied by the liquid phase. The remaining

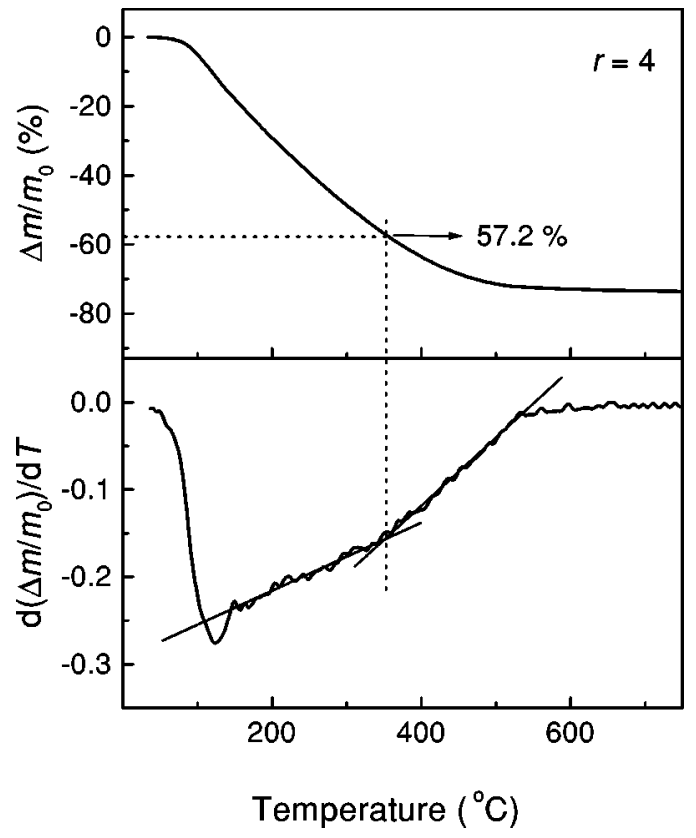

FIG. 6. Illustration of the obtaining of the parameter $\left(\Delta m / m_{0}\right)_{\xi}$ by the inflection point of the derivative of the TG curve showed for the sample with $r=4$. 
of the liquid phase is in the structure of the saturated clusters in such way to form a structure compatible with the corresponding mass fractal dimensionality.

The liquid phase outside of the clusters should evaporate with more facility in a thermogravimetric test than that inside of the clusters. Let $\left(\Delta m / m_{0}\right)_{\xi}$ be the mass loss fraction in which the liquid phase penetrates the clusters occupying a volume fraction $f_{\xi}$. Then

$$
\left(\Delta m / m_{0}\right)_{\xi}=\left(1-f_{\xi}\right) \rho_{\mathrm{L}} / \rho
$$

Figure 5 (bottom) shows the evaluation of $\left(\Delta m / m_{0}\right)_{\xi}$ as a function of the initial water/TMOS molar ratio. For instance, the evaluation of Eq. (11) for the sample with $r=4$ yields $\left(\Delta m / m_{0}\right)_{\xi}=0.563$.

Since the water phase within the clusters should present a greater resistance to evaporate than that outside the clusters, we expected a change in the evaporation rate at the instant in which the liquid phase penetrates the cluster, in a rapid evaporation process as in a TG test. Figure 6 detaches an inflection point in the first derivative of the weight loss curve for the sample with $r=4$. This inflection point occurs at $\left(\Delta m / m_{0}\right)_{\xi}=0.572$ in good agreement with the value 0.563 as obtained by SAXS. Figure 5 (bottom) shows the reasonable concordance between the values of $\left(\Delta m / m_{0}\right)_{\xi}$ as obtained by TG and by SAXS in all the studied range of the initial water/TMOS molar ratio.

\section{CONCLUSIONS}

The structure of the TMOS-derived sonogels, after about one year of aging in a hermetically sealed container, can be described as formed by mass fractal-like aggregates (clusters) of primary particles of silica, all imbibed in a liquid phase, a mixture of water and methanol resulting after a practically complete hydrolysis and polycondensation reaction. The mass fractal dimension of the clusters was found to be about 2.5, a value approximately independent of initial water/TMOS molar ratio. The characteristic length of the clusters increases with the initial water/TMOS molar ratio $(r)$ going from approximately $2.3 \mathrm{~nm}$ for $r=2$ to $4.5 \mathrm{~nm}$ for $r$ $=10$. The clusters are strongly aligned forming long chains of clusters in the case of $r=2$, while the distribution of size of the clusters becomes more wider for high water content.

Under a rapid evaporation process as in a thermogravimetric test, it is apparent that a change in the evaporation rate at the instant in which the liquid phase penetrates the cluster. In all cases of the studied range of the initial water/TMOS molar ratio, the values of weight loss fraction up to the liquid phase penetrates into the clusters as determined by TG are in reasonable agreement with the values of the volume fraction of the clusters as determined by SAXS.

\section{ACKNOWLEDGMENTS}

Research was partially supported by LNLS-National Synchrotron Light Laboratory (Brazil), and FAPESP (Brazil).
${ }^{1}$ C. J. Brinker and G. W. Scherer, Sol-Gel Science: The Physics and Chemistry of Sol-Gel Processing (Academic, San Diego, 1990).

${ }^{2}$ M. Tarasevich, Am. Ceram. Soc. Bull. 63, 500 (1984).

${ }^{3}$ C. Marlière, F. Despetis, P. Etienne, T. Woignier, P. Dieudonné, and J. Phalippou, J. Non-Cryst. Solids 285, 148 (2001).

${ }^{4}$ T. Woignier, J. Phalippou, R. Vacher, J. Pelous, and E. Courtens, J. Non-Cryst. Solids 121, 198 (1990).

${ }^{5}$ D. R. Vollet, D. A. Donatti, and A. Ibañez Ruiz, J. Non-Cryst. Solids 288, 81 (2001).

${ }^{6}$ D. A. Donatti, A. Ibañez Ruiz, and D. R. Vollet, J. Non-Cryst. Solids 292, 44 (2001).

${ }^{7}$ D. A. Donatti, A. Ibañez Ruiz, and D. R. Vollet, Ultrason.
Sonochem. 9, 133 (2002).

${ }^{8}$ D. A. Donatti, D. R. Vollet, and A. Ibañez Ruiz, J. Sol-Gel Sci. Technol. 18, 5 (2000).

${ }^{9}$ T. Freltoft, J. K. Kjems, and S. K. Sinha, Phys. Rev. B 33, 269 (1986).

${ }^{10}$ I. Krokovský, H. Urakawa, K. Kajiwara, and S. Kohjiya, J. NonCryst. Solids 231, 31 (1998).

${ }^{11}$ G. Porod, in Small Angle X-ray Scattering, edited by O. Glatter and O. Kratky (Academic, London, 1982).

${ }^{12}$ J. E. Martin and K. D. Keefer, Phys. Rev. A 34, 4988 (1986).

${ }^{13}$ R. Vacher, T. Woignier, J. Pelous, and E. Courtens, Phys. Rev. B 37, 6500 (1988). 